\title{
Topological Descriptors of Poly Propyl Ether Imine (PETIM) Dendrimers
}

\author{
Asfand Fahad ${ }^{1(\mathbb{D})}$, Muhammad Imran Qureshi ${ }^{1, *} \mathbb{( \mathbb { D }}$, Saima Noureen ${ }^{2}{ }^{(\mathbb{D})}$, Zahid Iqbal ${ }^{3}{ }^{\mathbb{D}}$, \\ Asim Zafar 1(D), Muhammad Ishaq 4(D) \\ 1 Department of Mathematics, COMSATS University Islamabad, Vehari 61100, Pakistan; asfandfahad1@yahoo.com \\ (A.F.); imranqureshi18@gmail.com (M.I.Q.); asimzafar@hotmail.com (A.Z.); \\ 2 Department of Mathematics, The Women University Multan, Multan 66000, Pakistan; saimanoureen2008@gmail.com \\ (S.N); \\ 3 Department of Mathematics and Statistics, Institute of Southern Punjab, Multan 66000, Pakistan; \\ 786zahidwarraich@gmail.com (Z.I.); \\ 4 School of Natural Sciences, National University of Science and Technology, Islamabad 44000, Pakistan; \\ ishaq_maths@yahoo.com (M.I.); \\ * Correspondence: imranqureshi18@gmail.com;
}

Scopus Author ID 57211399520

Received: 16.10.2020; Revised: 11.11.2020; Accepted: 12.11.2020; Published: 15.11.2020

Abstract: Topological descriptors, such as numeric number, a matrix, a sequence of numbers, or a polynomial, associated with a graph, are widely used to study physicochemical properties and QSAR/QSPR study. Several distances (eccentricity) and degree dependent topological indices are used to predict the bioactivity of several topological descriptors and polynomials of vastly used PETIM dendrimers.

Keywords: ABC index; GA index; Zagreb eccentric-connectivity index; eccentric-connectivity index; total-eccentricity index; PETIM dendrimers.

(C) 2020 by the authors. This article is an open-access article distributed under the terms and conditions of the Creative
Commons Attribution (CC BY) license (https://creativecommons.org/licenses/by/4.0/).

\section{Introduction}

In the modern era of computational chemistry, molecular structures are characterized by certain invariants associated with graphs, containing applications in QSAR/ASPR studies; see $[1,2]$. These invariants may associate a numeric number, a sequence of numbers, a matrix, a polynomial, or a drawing to a graph (or a molecular structure). Such values either possess similar trends or are identical for isomorphic graphs. One such revolutionary example of these invariants is a topological index (TI) of graphs (or molecular structure), which is sensitive to bonding, size, symmetry, shape, patterns, and heteroatoms' contents in a molecular structure. Therefore, the study of the TI yields the quantitative characterization of a certain molecular structure. Hence, evaluation and analysis of topological indices (TIs) of molecular structures are modern research trends, which are of significant importance in nanotechnology and theoretical chemistry. For a comprehensive literature review, the readers are referred to as [324].

On the other hand, dendrimers, comprising of the initial core and branches, possess several structured-based properties, particularly known for applications in medicines, have been vastly studied during the last two decades. A novel non-toxic dendrimer poly (propyl ether imine) (PETIM) has been synthesized and found suitable as a carrier for sustained drug ketoprofen delivery. Zidovudine (AZT), an antiretroviral drug for AIDS treatment (approved 
by FDA), when loaded with poly propyl ether imine (PETIM) dendrimer for its sustained drug delivery has been studied. The dendrimers are being vastly studied nowadays due to (potential) applications in several fields, such as biology, medicine, chemistry, physics, and engineering. For details, [25-33].

The standard notations have been used throughout the article, and most of the notations and definitions are taken from [1]. To describe this article's objective, we return to the TIs, which are mainly classified as degree and distance-based indices. We start with the following degree-based index:

The Randic connectivity index [34], introduced in 1975, for a graph $G$, it is defined by the formula:

$$
R(G)=\sum_{u v \in E(G)} \frac{1}{\sqrt{d_{u} d_{v}}}
$$

Here $d_{u}$ is representing the degree of vertex $u$. Its generalization, named the general product-connectivity index or general Randic connectivity index, is defined as follows:

$$
R_{\alpha}(G)=\sum_{u v \in E(G)}\left(d_{u} d_{v}\right)^{\alpha}
$$

Where $\alpha$ denotes any real number. Clearly, $R_{-\frac{1}{2}}(G)=R(G)$. The notion of the sumconnectivity index (SCI) was generalized as the general sum-connectivity index (GSCI) in [35] in the following way:

$$
\chi_{\alpha}(G)=\sum_{u v \in E(G)}\left(d_{u}+d_{v}\right)^{\alpha}
$$

SCI may be obtained from GSCI by taking $\alpha=-1 / 2$. These indices have been used to correlate the $\pi$-electron energy of benzenoid hydrocarbons [36]. The first generalized Zagreb index (FGZI) was introduced in [37] is defined as

$$
M_{\alpha}(G)=\sum_{u \in V(G)}\left(d_{u}\right)^{\alpha}
$$

Now, we briefly recall some other indices and their polynomials such as the eccentricconnectivity index (ECI) and its polynomial [38], the total-eccentricity index (TEI) and its polynomial [38], versions of Zagreb indices [39], ABC index [40], GA index [41], the fourth member of $\mathrm{ABC}$ index [42], the fifth version of GA index and (GA5) [43], respectively. The symbol $\varepsilon(u)$ represents the eccentricity of the vertex $u$ in a graph. $S_{u}$ represent the degree sum of neighbor vertices for $u$.

$$
\begin{gathered}
\xi(G)=\sum_{u \in V(G)} \varepsilon(u) d_{u} \\
\operatorname{ECP}(G, y)=\sum_{u \in V(G)} d_{u} y^{\varepsilon(u)} \\
\varsigma(G)=\sum_{u \in V(G)} \varepsilon(u) \\
\operatorname{TECP}(G, y)=\sum_{u \in V(G)} y^{\varepsilon(u)} \\
M_{1}^{* *}(G)=\sum_{u \in V(G)}(\varepsilon(u))^{2} \\
M_{2}^{*}(G)=\sum_{u v \in E(G)} \varepsilon(u) \varepsilon(v)
\end{gathered}
$$


(10)

$$
\begin{aligned}
A B C(G) & =\sum_{u v \in E(G)} \sqrt{\frac{d_{u}+d_{v}-2}{d_{u} d_{v}}} \\
G A(G) & =\sum_{u v \in E(G)} \frac{2 \sqrt{d_{u} d_{v}}}{d_{u}+d_{v}} \\
A B C_{4}(G) & =\sum_{u v \in E(G)} \sqrt{\frac{S_{u}+S_{v}-2}{S_{u} S_{v}}} \\
G A_{5}(G) & =\sum_{u v \in E(G)} \frac{2 \sqrt{S_{u} S_{v}}}{S_{u}+S_{v}}
\end{aligned}
$$

In this article, we compute and analyze several degrees and distance-based TIs for the PETIM Dendrimer. Based on the degree and distance-dependent descriptors, this article's results have been divided into the following section.

\section{Materials and Methods}

Let $D(n)$ denotes the molecular graph of PETIM dendrimers, where $n$ represents the generation stage of $D(n)$. The graph for $D(3)$ has been shown in Figure 1. From the figure, it is clear that $|V(D(n))|=3 \times 2^{n+3}-23$ and $|E(D(n))|=24\left(2^{n}-1\right)$.

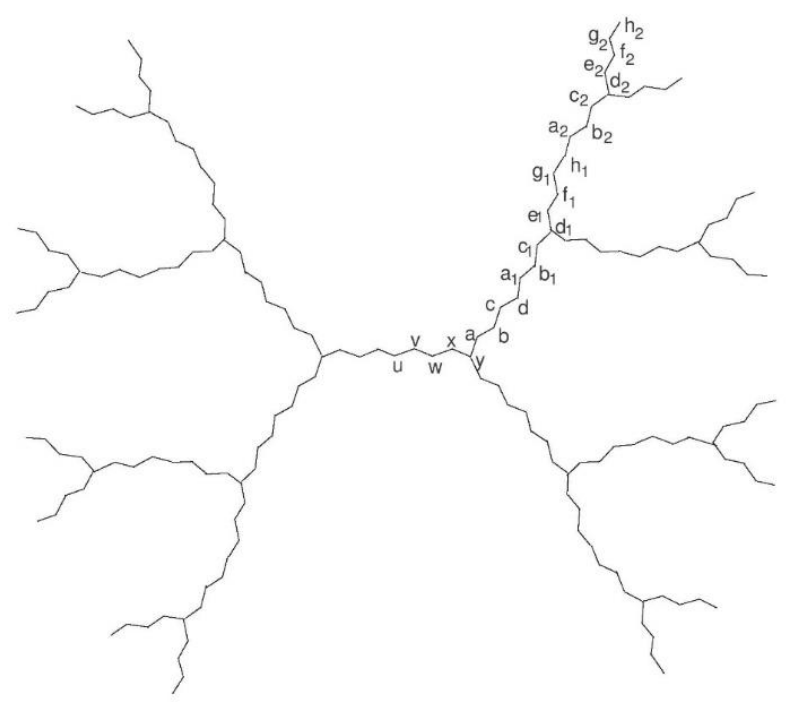

Figure 1. The Third generation stage of $D(n)$.

\section{Results and Discussion}

\subsection{Degree based descriptors of PETIM dendrimers.}

The main objective of this section is to $\operatorname{compute}_{\alpha}(D(n)), R_{\alpha}(D(n))$, $\chi_{\alpha}(G D(n)), A B C(D(n)), G A(D(n)), A B C_{4}(D(n))$ and $G A_{5}(D(n))$ for any size $n$ and any real number $\alpha$. The following table will be useful for the computation of TIs.

Table 1. The edge partition of graph $D(n)$ based on the degree of end vertices of each edge.

\begin{tabular}{c|c}
$\left(\boldsymbol{d}_{\boldsymbol{u}}, \boldsymbol{d}_{\boldsymbol{v}}\right)$ where $\boldsymbol{u} \boldsymbol{v} \in \boldsymbol{E}(\boldsymbol{D}(\boldsymbol{n}))$ & Number of edges \\
\hline$(1,2)$ & $2^{n+1}$ \\
\hline$(2,2)$ & $2\left(2^{n+3}-9\right)$ \\
\hline$(2,3)$ & $6\left(2^{n}-1\right)$
\end{tabular}


Theorem 3.1: For $D(n)$, we have the following

1) $M_{\alpha}(D(n))=2^{n+1}\left(1+2^{\alpha} \times 10+3^{\alpha}\right)-21 \times 2^{\alpha}-2 \times 3^{\alpha}$.

2) $R_{\alpha}(D(n))=2^{\alpha+1}\left(2^{n}+3^{\alpha+1}\left(2^{n}-1\right)+2^{\alpha}\left(2^{n+3}-9\right)\right)$.

3) $\chi_{\alpha}(D(n))=2^{n+1}\left(3^{\alpha}+4^{\alpha}\left(8-2^{-n} \times 9\right)+3 \times 5^{\alpha}\left(1-2^{-n}\right)\right)$.

Proof: In $D(n)$, there are $3 \times 2^{n+3}-23$ vertices, distributed as $2^{n+1}, 5 \times 2^{n+2}-21$ and $2^{n+1}-2$ vertices having degrees 1,2 , and 3 , respectively. By replacing these values in the formula for $M_{\alpha}(G)$, we get the required values. Clearly, $|E(D(n))|=24\left(2^{n}-1\right)$; consequently, we get the edge partition (based on the degree of vertices), shown in Table 1. By using Table 1 in the formulas for $R_{\alpha}(G)$ and $\chi_{\alpha}(G)$, we obtain the required results.

Theorem 3.2: For $D(n)$, we have

$$
A B C(D(n))=\frac{1}{\sqrt{2}}\left(24\left(2^{n}-1\right)\right)
$$

Proof: From Table 1 and (10), we get

$$
A B C(D(n))=2^{n+1} \sqrt{\frac{1+2-2}{1 \times 2}}+2\left(2^{n+3}-9\right) \sqrt{\frac{2+2-2}{2 \times 2}}+6\left(2^{n}-1\right) \sqrt{\frac{2+3-2}{2 \times 3}}
$$

which yields

$$
A B C(D(n))=\frac{1}{\sqrt{2}}\left(24\left(2^{n}-1\right)\right)
$$

Theorem 3.3: For $D(n)$, we have

$$
G A(D(n))=2^{n+2}\left(\frac{\sqrt{2}}{3}+4+\frac{3 \sqrt{6}}{5}\right)-6\left(3+\frac{2 \sqrt{6}}{5}\right)
$$

Proof: From equation (11) and Table 1, we get

$$
G A(D(n))=2^{n+1} \frac{2 \sqrt{1 \times 2}}{1+2}+2\left(2^{n+3}-9\right) \frac{2 \sqrt{2 \times 2}}{2+2}+6\left(2^{n}-1\right) \frac{2 \sqrt{2 \times 3}}{2+3}
$$

which upon simplification gives

$$
G A(D(n))=2^{n+2}\left(\frac{\sqrt{2}}{3}+4+\frac{3 \sqrt{6}}{5}\right)-6\left(3+\frac{2 \sqrt{6}}{5}\right)
$$

Now by using Table 2, we compute $A B C_{4}(D(n))$ and $G A_{5}(D(n))$ in the next theorems.

Table 2. The edge partition of graph $D(n)$ based on the degree sum of neighbor vertices of end vertices of each edge.

\begin{tabular}{c|c}
$\left(\boldsymbol{S}_{\boldsymbol{u}}, \boldsymbol{S}_{\boldsymbol{v}}\right)$ where $\boldsymbol{u} \boldsymbol{v} \in \boldsymbol{E}(\boldsymbol{D}(\boldsymbol{n}))$ & Number of edges \\
\hline$(2,3)$ & $2^{n+1}$ \\
\hline$(3,4)$ & $2^{n+1}$ \\
\hline$(4,4)$ & 4 \\
\hline$(4,5)$ & $14 \times 2^{n}-22$ \\
\hline$(5,6)$ & $6\left(2^{n}-1\right)$
\end{tabular}

Theorem 3.4: For $D(n)$, we have

$$
A B C_{4}(D(n))=2^{n}\left(\sqrt{2}+\frac{\sqrt{5}}{\sqrt{3}}+\frac{6 \sqrt{3}}{\sqrt{10}}+\frac{7 \sqrt{7}}{\sqrt{5}}\right)-\frac{6 \sqrt{3}}{\sqrt{10}}-\frac{11 \sqrt{7}}{\sqrt{5}}+\sqrt{6}
$$

Proof: From Table 2 and equation (12), we obtain

$$
A B C_{4}(D(n))=2^{n+1} \sqrt{\frac{2+3-2}{2 \times 3}}+2^{n+1} \sqrt{\frac{3+4-2}{3 \times 4}}+4 \sqrt{\frac{4+4-2}{4 \times 4}}
$$


By simplifying, we get

$$
+\left(14 \times 2^{n}-22\right) \sqrt{\frac{4+5-2}{4 \times 5}}+6\left(2^{n}-1\right) \sqrt{\frac{5+6-2}{5 \times 6}}
$$

$$
A B C_{4}(D(n))=2^{n}\left(\sqrt{2}+\frac{\sqrt{5}}{\sqrt{3}}+\frac{6 \sqrt{3}}{\sqrt{10}}+\frac{7 \sqrt{7}}{\sqrt{5}}\right)-\frac{6 \sqrt{3}}{\sqrt{10}}-\frac{11 \sqrt{7}}{\sqrt{5}}+\sqrt{6}
$$

Theorem 3.5: For $D(n)$, we have

$$
G A_{4}(D(n))=2^{n+3}\left(\frac{\sqrt{6}}{10}+\frac{7 \sqrt{5}}{9}+\frac{\sqrt{3}}{7}+\frac{3 \sqrt{30}}{22}\right)-4\left(1-\frac{22 \sqrt{5}}{9}-\frac{3 \sqrt{30}}{11}\right)
$$

Proof: We find the edge partition of the $D(n)$ based on the degree sum of vertices lying at unit distance from end vertices of each edge, as shown in Table 2. By using Table 2 and equation (13), we obtain

$$
\begin{aligned}
G A_{5}(D(n))= & 2^{n+1} \frac{2 \sqrt{2 \times 3}}{2+3}+2^{n+1} \frac{2 \sqrt{3 \times 4}}{3+4}+4 \frac{2 \sqrt{4 \times 4}}{4+4}+\left(14 \times 2^{n}-22\right) \frac{2 \sqrt{4 \times 5}}{4+5} \\
& +6\left(2^{n}-1\right) \frac{2 \sqrt{5 \times 6}}{5+6}
\end{aligned}
$$

After some calculations, we have:

$$
G A_{4}(D(n))=2^{n+3}\left(\frac{\sqrt{6}}{10}+\frac{7 \sqrt{5}}{9}+\frac{\sqrt{3}}{7}+\frac{3 \sqrt{30}}{22}\right)-4\left(1-\frac{22 \sqrt{5}}{9}-\frac{3 \sqrt{30}}{11}\right)
$$

\subsection{Eccentricity based descriptors of PETIM dendrimers.}

This section is devoted to the computation and analysis of several eccentricity-based topological descriptors and polynomial of PETIM dendrimer.

Table 3. The core vertices with eccentricity, frequency, and degree for $n \geq 1$.

\begin{tabular}{c|c|c|c} 
Representative & Degree & Eccentricity & Frequency \\
\hline u & 2 & $8 n$ & 1 \\
\hline v & 2 & $8 n+1$ & 2 \\
\hline w & 2 & $8 n+2$ & 2 \\
\hline x & 2 & $8 n+3$ & 2 \\
\hline y & 3 & $8 n+4$ & 2
\end{tabular}

Table 4. The first generation vertices along with eccentricity, frequency, and degree for $n \geq 1$.

\begin{tabular}{c|c|c|c} 
Representative & Degree & Eccentricity & Frequency \\
\hline $\mathrm{a}$ & 2 & $8 n+5$ & 4 \\
\hline $\mathrm{b}$ & 2 & $8 n+6$ & 4 \\
\hline $\mathrm{c}$ & 2 & $8 n+7$ & 4 \\
\hline $\mathrm{d}$ when $n=1$ & 1 & $8 n+8$ & 4 \\
\hline $\mathrm{d}$ when $n \geq 2$ & 2 & $8 n+8$ & 4
\end{tabular}

Table 5. The second-generation vertices, along with eccentricity, frequency, degree for $n \geq 2$.

\begin{tabular}{c|c|c|c} 
Representative & Degree & Eccentricity & Frequency \\
\hline$a_{i}$ & 2 & $8 n+8 i+1$ & $2^{i+1}$ \\
\hline$b_{i}$ & 2 & $8 n+8 i+2$ & $2^{i+1}$ \\
\hline$c_{i}$ & 2 & $8 n+8 i+3$ & $2^{i+1}$ \\
\hline$d_{i}$ & 3 & $8 n+8 i+4$ & $2^{i+1}$ \\
\hline$e_{i}$ & 2 & $8 n+8 i+5$ & $2^{i+2}$ \\
\hline$f_{i}$ & 2 & $8 n+8 i+6$ & $2^{i+2}$ \\
\hline$g_{i}$ & 2 & $8 n+8 i+7$ & $2^{i+2}$ \\
\hline$h_{i}$ when $i \neq n-1$ & 2 & $8 n+8 i+8$ & $2^{i+2}$ \\
\hline$h_{i}$ when $i=n-1$ & 1 & $16 n$ & $2^{n+1}$
\end{tabular}

We start by computing the $\xi(D(n))$ in the following theorem. 
Theorem 3.6: For $D(n)$, the $E C I$, in term of $n$ is given by

$$
\xi(D(n))=\left\{\begin{array}{cc}
608, & n=1 \\
32\left(24 n \times 2^{n}-12 n-17 \times 2^{n}+17\right) & n \geq 2
\end{array}\right.
$$

Proof: The symmetrical structure of $D(n)$ facilitates us to use its one branch, see Figure 1 . We take representatives sets from a set of vertices with the same degree and eccentricity for the core, first-generation, and the remaining generations. The representatives set of the core vertices are labeled by $u, v, w, x$, and $y$. This set of representatives with their eccentricities, frequencies, and degrees of occurrence is given in Table 3 . The representatives set of vertices introduced in the first generation are labeled by $a, b, c$, and $d$. This set of representatives with their eccentricities, frequencies, and degrees of occurrence is given in Table 4. The representatives set of vertices introduced in the remaining generations are labeled $a_{i}, b_{i}, c_{i}, d_{i}, e_{i}, f_{i}, g_{i}, h_{i}$, where $1 \leq i \leq n-1$. This set of representatives with their eccentricities, frequencies, and degrees of occurrence is described in Table 5.

By using Table 3 and Table 4, we have

$$
\begin{aligned}
\xi(D(n))= & \sum_{u \in V(D(n))} \varepsilon(u) d_{u} \\
& =(1 \times 2) 8+(2 \times 2)(8+1)+(2 \times 2)(8+2)+(2 \times 2)(8+3) \\
& +(2 \times 3)(8+4)+(2 \times 4)(8+5)+(2 \times 4)(8+6)+(2 \times 4)(8+7) \\
& +(1 \times 4)(8+8)=608 .
\end{aligned}
$$

By using Table 3, Table 4, and Table 5, the eccentric-connectivity index of $D(n)$ for $n \geq 2$ can be written as follows:

$$
\begin{aligned}
\xi(D(n))= & \sum_{u \in V(D(n))} \varepsilon(u) d_{u} \\
& =(1 \times 2) 8 n+(2 \times 2)(8 n+1)+(2 \times 2)(8 n+2)+(2 \times 2)(8 n+3) \\
& +(2 \times 3)(8 n+4)+(2 \times 4)(8 n+5)+(2 \times 4)(8 n+6) \\
& +(2 \times 4)(8 n+7)+(2 \times 4)(8 n+8)+2^{n+1}(16 n) \\
& +\sum_{i=1}^{n-1} 2^{i+1}(2(8 n+8 i+1)+2(8 n+8 i+2)+2(8 n+8 i+3) \\
& +3(8 n+8 i+4)) \\
& +\sum_{i=1}^{n-1} 2 \times 2^{i+2}((8 n+8 i+5)+(8 n+8 i+6)+(8 n+8 i+7)) \\
& +\sum_{i=1}^{n-2} 2^{i+2} 2(8 n+8 i+8) .
\end{aligned}
$$

After some calculations, we get

$$
\xi(D(n))=32\left(24 n \times 2^{n}-12 n-17 \times 2^{n}+17\right)
$$

Corollary 3.7: For $D(n)$, the eccentric-connectivity polynomials for $n=1$ and $n \geq 2$ are given by

$\operatorname{TECP}(D(1), y)=2 y^{8}\left(2 y^{8}+4 y^{7}+4 y^{6}+4 y^{5}+3 y^{4}+2 y^{3}+2 y^{2}+2 y+1\right)$, $\operatorname{TECP}(D(n), y)=2 y^{8 n}\left(4 y^{8}+4 y^{7}+4 y^{6}+4 y^{5}+3 y^{4}+2 y^{3}+2 y^{2}+2 y+2^{n} y^{8 n}+1\right)$ $+\frac{2\left(4 y^{6}+4 y^{5}+4 y^{4}+3 y^{3}+2 y^{2}+2 y+2\right) y^{8 n+1}\left(2^{n} y^{8 n}-2 y^{8}\right)+4 y^{8 n}\left(2^{n} y^{8 n}-4 y^{16}\right)}{2 y^{8}-1}$ 
Theorem 3.8: For $D(n)$, the $\varsigma(D(n))$ is given by

$$
\varsigma(D(n))=\left\{\begin{array}{cc}
324, & n=1 \\
4\left(96 n \times 2^{n}-46 n-65 \times 2^{n}+65\right) & n \geq 2
\end{array}\right.
$$

Proof: If $n=1$, Table 3 and Table 4 yield

$$
\begin{aligned}
s(D(1))= & \sum_{u \in V(D(n))} \varepsilon(u) \\
& =8+2((8+1)+(8+2)+(8+3)+(8+4)) \\
& +4((8+5)+(8+6)+(8+7)+(8+8))=324
\end{aligned}
$$

For $n \geq 2$, from Table 3-5, we have

$$
\begin{aligned}
\varsigma(D(n))= & \sum_{u \in V(D(n))} \varepsilon(u) \\
& =8 n+2((8 n+1)+(8 n+2)+(8 n+3)+(8 n+4)) \\
& +4((8 n+5)+(8 n+6)+(8 n+7)+(8 n+8))+2^{n+1}(16 n) \\
& +\sum_{i=1}^{n-1} 2^{i+1}((8 n+8 i+1)+(8 n+8 i+2)+(8 n+8 i+3) \\
& +(8 n+8 i+4)) \\
& +\sum_{i=1}^{n-1} 2^{i+2}((8 n+8 i+5)+(8 n+8 i+6)+(8 n+8 i+7)) \\
& +\sum_{i=1}^{n-2} 2^{i+2}(8 n+8 i+8) .
\end{aligned}
$$

Which upon simplification give

$$
=4\left(96 n \times 2^{n}-46 n-65 \times 2^{n}+65\right)
$$

Corollary 3.9: For $D(n)$, the total eccentric-connectivity polynomials for $n=1$ and $n \geq 2$ are given by

$$
\begin{gathered}
\operatorname{TECP}(D(1), y)=y^{8}\left(4 y^{8}+4 y^{7}+4 y^{6}+4 y^{5}+2 y^{4}+2 y^{3}+2 y^{2}+2 y+1\right), \\
\operatorname{TECP}(D(n), y)=y^{8 n}\left(4 y^{8}+4 y^{7}+4 y^{6}+4 y^{5}+2 y^{4}+2 y^{3}+2 y^{2}+2 y+2^{n+1} y^{8 n}+1\right) \\
+\frac{2\left(2 y^{6}+2 y^{5}+2 y^{4}+y^{3}+y^{2}+y+1\right) y^{8 n+1}\left(2^{n} y^{8 n}-2 y^{8}\right)+2 y^{8 n}\left(2^{n} y^{8 n}-4 y^{16}\right)}{2 y^{8}-1}
\end{gathered}
$$

Theorem 3.10: For $D(n)$, the second Zagreb eccentricity index is given by

$$
\begin{array}{ll}
M_{1}^{* *}(D(n)) & 4340 \\
=\left\{\begin{array}{cc}
\left.436 n^{2} \times 2^{n}+1040 n-368 n^{2}-2080 n \times 2^{n}+1501 \times 2^{n}-1501\right) & n \geq 2 \\
4\left(1536 n^{2}\right.
\end{array}\right.
\end{array}
$$

Proof: If $n=1$, Tables 3-4 yield

$$
\begin{aligned}
M_{1}^{* *}(D(1))= & \sum_{u \in V(D(1))}[\varepsilon(u)]^{2} \\
& =8^{2}+2\left((8+1)^{2}+(8+2)^{2}+(8+3)^{2}+(8+4)^{2}\right) \\
& +4\left((8+5)^{2}+(8+6)^{2}+(8+7)^{2}+(8+8)^{2}\right)=4340
\end{aligned}
$$

From Tables 3-5, we have 


$$
\begin{aligned}
M_{1}^{* *}(D(n))= & \sum_{u \in V(D(n))}[\varepsilon(u)]^{2} \\
& =(8 n)^{2}+2\left((8 n+1)^{2}+(8 n+2)^{2}+(8 n+3)^{2}+(8 n+4)^{2}\right) \\
& +4\left((8 n+5)^{2}+(8 n+6)^{2}+(8 n+7)^{2}+(8 n+8)^{2}\right)+2^{n+1}(16 n)^{2} \\
& +\sum_{i=1}^{n-1} 2^{i+1}\left((8 n+8 i+1)^{2}+(8 n+8 i+2)^{2}+(8 n+8 i+3)^{2}\right. \\
& \left.+(8 n+8 i+4)^{2}\right) \\
& +\sum_{i=1}^{n-1} 2^{i+2}\left((8 n+8 i+5)^{2}+(8 n+8 i+6)^{2}+(8 n+8 i+7)^{2}\right) \\
& +\sum_{i=1}^{n-2} 2^{i+2}(8 n+8 i+8)^{2} .
\end{aligned}
$$

After some calculations, we obtain

$$
=4\left(1536 n^{2} \times 2^{n}+1040 n-368 n^{2}-2080 n \times 2^{n}+1501 \times 2^{n}-1501\right)
$$

Table 6. The edge partition of $D(n)$ with respect to the representatives of pairs of end vertices and their frequencey of occurrence.

\begin{tabular}{c|c|c} 
Representative & Eccentricity & Frequency \\
\hline$[u, v]$ & {$[8 n, 8 n+1]$} & 2 \\
\hline$[v, w]$ & {$[8 n+1,8 n+2]$} & 2 \\
\hline$[w, x]$ & {$[8 n+2,8 n+3]$} & 2 \\
\hline$[x, y]$ & {$[8 n+3,8 n+4]$} & 2 \\
\hline$[y, a]$ & {$[8 n+4,8 n+5]$} & 4 \\
\hline$[a, b]$ & {$[8 n+5,8 n+6]$} & 4 \\
\hline$[b, c]$ & {$[8 n+6,8 n+7]$} & 4 \\
\hline$[c, d]$ & {$[8 n+7,8 n+8]$} & 4 \\
\hline$\left[d, a_{1}\right]$ & {$[8 n+8,8 n+9]$} & 4 \\
\hline$\left[a_{i}, b_{i}\right]$ & {$[8 n+8 i+1,8 n+8 i+2]$} & $2^{i+1}$ \\
\hline$\left[b_{i}, c_{i}\right]$ & {$[8 n+8 i+2,8 n+8 i+3]$} & $2^{i+1}$ \\
\hline$\left[c_{i}, d_{i}\right]$ & {$[8 n+8 i+3,8 n+8 i+4]$} & $2^{i+2}$ \\
\hline$\left[d_{i}, e_{i}\right]$ & {$[8 n+8 i+4,8 n+8 i+5]$} & $2^{i+2}$ \\
\hline$\left[e_{i}, f_{i}\right]$ & {$[8 n+8 i+5,8 n+8 i+6]$} & $2^{i+2}$ \\
\hline$\left[f_{i}, g_{i}\right]$ & {$[8 n+8 i+6,8 n+8 i+7]$} & $2^{i+2}$ \\
\hline$\left[g_{i}, h_{i}\right]$ & {$[8 n+8 i+7,8 n+8 i+8]$} & $2^{i+2}$
\end{tabular}

Theorem 3.11: For $D(n)$, the third Zagreb eccentricity index is given by

$$
M_{2}^{*}(D(n))=\left\{\begin{array}{cc}
3960 & n=1 \\
8\left(768 n^{2} \times 2^{n}+783 \times 2^{n}+544 n-192 n^{2}-1088 n \times 2^{n}-783\right) & n \geq 2
\end{array}\right.
$$

Proof: The edge partition of $D(n)$ with respect to the representatives of pairs of end vertices and their frequency of occurrence is shown in Table 6 . The eccentricities are taken from Tables 3, 4, and 5. From Table 6, we compute the third Zagreb eccentricity index of $D(n)$ for $n=1$ as follows:

$$
\begin{aligned}
M_{2}^{*}(D(1))= & \sum_{u v \in E(D(1))} \varepsilon(u) \varepsilon(v) \\
& =2(8(8+1)+(8+1)(8+2)+(8+2)(8+3)+(8+3)(8+4)) \\
& +4((8+4)(8+5)+(8+5)(8+6)+(8+6)(8+7)+(8+7)(8+8)) \\
& =3960
\end{aligned}
$$


From Table 6, we compute the third Zagreb eccentricity index of $D(n)$ for $n \geq 2$ as follows:

$$
\begin{aligned}
M_{2}^{*}(D(n))= & \sum_{u v \in E(D(n))} \varepsilon(u) \varepsilon(v) \\
= & 2(8 n(8 n+1)+(8 n+1)(8 n+2)+(8 n+2)(8 n+3) \\
& +(8 n+3)(8 n+4)) \\
& +4((8 n+4)(8 n+5)+(8 n+5)(8 n+6)+(8 n+6)(8 n+7) \\
& +(8 n+7)(8 n+8)+(8 n+8)(8 n+9)) \\
& +\sum_{i=1}^{n-1} 2^{i+1}((8 n+8 i+1)(8 n+8 i+2)+(8 n+8 i+2)(8 n+8 i+3) \\
& +(8 n+8 i+3)(8 n+8 i+4)) \\
& +\sum_{i=1}^{n-1} 2^{i+2}((8 n+8 i+4)(8 n+8 i+5)+(8 n+8 i+5)(8 n+8 i+6) \\
& +(8 n+8 i+6)(8 n+8 i+7)+(8 n+8 i+7)(8 n+8 i+8)) \\
& +\sum_{i=1}^{n-2} 2^{i+2}(8 n+8 i+8)(8 n+8 i+9)
\end{aligned}
$$

After some calculations, we have

$$
=8\left(768 n^{2} \times 2^{n}+783 \times 2^{n}+544 n-192 n^{2}-1088 n \times 2^{n}-783\right)
$$

\section{Conclusions}

In this paper, we have computed several distances and degree dependent topological descriptors for vastly studied dendrimers known as PETIM. These results may help understand the behavior of some properties of these dendrimers. We have computed the values for any generation size $n$ of the PETIM, but further targets in this regard may be achieved with the collaboration between pharmacists and mathematicians (or by using the values obtained in this work).

\section{Funding}

This research received no external funding.

\section{Acknowledgments}

All the authors are thankful to their respective institutions.

\section{Conflicts of Interest}

The authors declare no conflict of interest.

\section{References}

1. Harary, F. Graph Theory. Addison Wesley Publishing Company. Reading, MA, USA, 1969.

2. Trinajstić, N. Chemical graph theory, $2^{\text {nd }}$ ed.; CRC Press, Boca Raton: Florida, 1992.

3. Gao, W.; Iqbal, Z.; Ishaq, M.; Aslam, A.; Aamir, M.; Binyamin, M.A. Bounds on Topological Descriptors of the Corona Product of \$F\$ -Sum of Connected Graphs. IEEE Access 2019, 7, 26788-26796, https://doi.org//10.1109/ACCESS.2019.2900061. 
4. Gao, W.; Iqbal, Z.; Ishaq, M.; Sarfraz, R.; Aamir, M.; Aslam, A. On eccentricity-based topological indices study of a class of porphyrin-cored dendrimers. Biomolecules 2018, 8, 71, https://doi.org/10.3390/biom8030071.

5. Gao, Y.; Zhu, E.; Shao, Z.; Gutman, I.; Klobučar, A. Total domination and open packing in some chemical graphs. J. Math. Chem. 2018, 56, 1481-1492, https://doi.org/10.1007/s10910-018-0877-6.

6. Yang, H.; Imran, M.; Akhter, S.; Iqbal, Z.; Siddiqui, M.K. On Distance-Based Topological Descriptors of Subdivision Vertex-Edge Join of Three Graphs. IEEE Access 2019, 7, 143381-143391, https://doi.org/10.1109/ACCESS.2019.2944860.

7. Iqbal, Z.; Aslam, A.; Ishaq, M.; Gao, W. The Edge Versions of Degree-Based Topological Descriptors of Dendrimers. J. Cluster Sci. 2020, 31, 445-452, https://doi.org/10.1007/s10876-019-01658-w.

8. Zheng, J.; Iqbal, Z.; Fahad, A.; Zafar, A.; Aslam, A.; Qureshi, M.I.; Irfan, R. Some eccentricity-based topological indices and polynomials of poly (EThyleneAmidoAmine)(PETAA) dendrimers. Processes 2019, 7, 433, https://doi.org/10.3390/pr7070433.

9. Gao, W.; Akhter, S.; Iqbal, Z.; Qasim, M.; Aslam, A. The Topological Aspects of Phthalocyanines and $\begin{array}{lllll}\text { Porphyrins Dendrimers. } & \text { IEEE } & & \end{array}$ https://doi.org/10.1109/ACCESS.2020.3023658

10. Zahid, I.; Muhammad, I.; Adnan, A.; Wei, G. On eccentricity-based topological descriptors of water-soluble dendrimers. Zeitschrift für Naturforschung C 2019, 74, 25-33, https://doi.org/10.1515/znc-2018-0123.

11. Luo, L.; Dehgardi, N.; Fahad, A. Lower Bounds on the Entire Zagreb Indices of Trees. Discrete Dynamics in Nature and Society 2020, 2020, https://doi.org/10.1155/2020/8616725.

12. Zhang, X.; Raza, A.; Fahad, A.; Jamil, M.K.; Chaudhry, M.A.; Iqbal, Z. On Face Index of Silicon Carbides. Discrete Dynamics in Nature and Society 2020, 2020, https://doi.org/10.1155/2020/6048438.

13. Ye, A.; Javed, A.; Jamil, M.K.; Abdul Sattar, K.; Aslam, A.; Iqbal, Z.; Fahad, A. On Computation of Face Index of Certain Nanotubes. Discrete Dynamics in Nature and Society 2020, 2020, https://doi.org/10.1155/2020/3468426.

14. Imran, M.; Nawaz, T.; Malik, M.A.; Jamil, M.K.; Hayat, S. On topological properties of boron and boron- $\alpha$ nanotubes. Mathematical methods in applied sciences 2020, 1-15, https://doi.org/10.1002/mma.6808.

15. Zhao, D.; Iqbal, Z.; Irfan, R.; Chaudhry, M.A.; Ishaq, M.; Jamil, M.K.; Fahad, A. Comparison of Irregularity Indices of Several Dendrimers Structures. Processes 2019, 7, https://doi.org/10.3390/pr7100662.

16. Wei, C.-C.; Ali, H.; Binyamin, M.A.; Naeem, M.N.; Liu, J.-B. Computing Degree Based Topological Properties of Third Type of Hex-Derived Networks. Mathematics 2019, 7, https://doi.org/10.3390/math7040368.

17. Ali, H.; Binyamin, M.A.; Shafiq, M.K.; Gao, W. On the Degree-Based Topological Indices of Some Derived Networks. Mathematics 2019, 7, https://doi.org/10.3390/math7070612.

18. Iqbal, Z.; Aslam, A.; Ishaq, M.; Aamir, M. Characteristic study of irregularity measures of some nanotubes. Canadian Journal of Physics 2019, 97, 1125-1132, https://doi.org/10.1139/cjp-2018-0619.

19. Gao, W.; Aamir, M.; Iqbal, Z.; Ishaq, M.; Aslam, A. On Irregularity Measures of Some Dendrimers Structures. Mathematics 2019, 7, https://doi.org/10.3390/math7030271.

20. Ye, A.; Qureshi, M.I.; Fahad, A.; Aslam, A.; Jamil, M.K.; Zafar, A.; Irfan, R. Zagreb Connection Number Index of Nanotubes and Regular Hexagonal Lattice. Open Chemistry 2019, 17, 75-80, https://doi.org/10.1515/chem-2019-0007.

21. Jalil, A.; Javed, A.; Jamil, M.K.; Alaeiyan, M.; Farahani, M.R. Topological properties of four types of porphyrin dendrimers. Proyecciones Journal of Mathematics 2020, 39(4), 979-993, DOI: 10.22199/issn.0717-6279-2020-04-0061.

22. Alfuraidan, M.R.; Imran, M.; Jamil, M.K.; Vetrik, T. General multiplicative Zagreb indices of graphs with indices. IEEE Access 2020, 118725 - 118731, DOI: 10.1109/ACCESS.2020.3005040.

23. Mojdeh, D.A.; Habibi, M.; Badakhshian, L.; Rao, Y. Zagreb indices of trees, unicyclic and bicyclic graphs with given (total) domination. IEEE Access 2019, 7, 94143-94149, DOI: 10.1109/ACCESS.2019.2927288.

24. Liu, J.B.; Javaid, M.; Awais, H.M. Computing Zagreb indices of the subdivision-related generalized operations of graphs, IEEE Access 2019, 7, 105479-105488, DOI: 10.1109/ACCESS.2019.2932002.

25. Adronov, A.; Fréchet, J.M.J. Light-harvesting dendrimers. Chem. Commun. 2000, 1701-1710, https://doi.org/10.1039/B005993P.

26. Ballauff, M. Structure of dendrimers in dilute solution. In Dendrimers III, Springer: 2001; 177-194.

27. Bosman, A.W.; Janssen, H.M.; Meijer, E.W. About Dendrimers: Structure, Physical Properties, and Applications. Chem. Rev. 1999, 99, 1665-1688, https://doi.org/10.1021/cr970069y.

28. Jain, S.; Kaur, A.; Puri, R.; Utreja, P.; Jain, A.; Bhide, M.; Ratnam, R.; Singh, V.; Patil, A.S.; Jayaraman, N.; Kaushik, G.; Yadav, S.; Khanduja, K.L. Poly propyl ether imine (PETIM) dendrimer: A novel non-toxic dendrimer for sustained drug delivery. Eur. J. Med. Chem. 2010, 45, 4997-5005, https://doi.org/10.1016/j.ejmech.2010.08.006.

29. Krishna, T.R.; Jayaraman, N. Synthesis of Poly(propyl ether imine) Dendrimers and Evaluation of Their Cytotoxic Properties. The Journal of Organic Chemistry 2003, 68, 9694-9704, https://doi.org/10.1021/jo035072y. 
30. Liang, Z.; Gong, T.; Sun, X.; Tang, J.Z.; Zhang, Z. Chitosan oligomers as drug carriers for renal delivery of zidovudine. Carbohydr. Polym. 2012, 87, 2284-2290, https://doi.org/10.1016/j.carbpol.2011.10.060.

31. Naka, K.; Tanaka, Y.; Chujo, Y. Effect of Anionic Starburst Dendrimers on the Crystallization of CaCO3 in Aqueous Solution: Size Control of Spherical Vaterite Particles. Langmuir 2002, 18, 3655-3658, https://doi.org/10.1021/la011345d.

32. Svenson, S.; Tomalia, D.A. Dendrimers in biomedical applications-reflections on the field. Adv. Drug Del. Rev. 2012, 64, 102-115, https://doi.org/10.1016/j.addr.2012.09.030.

33. Suresh, R.; Singh, C.; Rewar, P. Dendrimers as carriers and its application in therapy. Int. J. Anal. Pharm. Biomed. Sci 2015, 4, 15-23.

34. Randic, M. Characterization of molecular branching. J. Am. Chem. Soc. 1975, 97, 6609-6615, https://doi.org/10.1021/ja00856a001.

35. Zhou, B.; Trinajstić, N. On general sum-connectivity index. J. Math. Chem. 2010, 47, 210-218, https://doi.org/10.1007/s10910-009-9542-4.

36. Lučić, B.; Trinajstić, N.; Zhou, B. Comparison between the sum-connectivity index and product-connectivity index for benzenoid hydrocarbons. Chem. Phys. Lett. 2009, 475, 146-148, https://doi.org/10.1016/j.cplett.2009.05.022.

37. Li, X.; Zhao, H. Trees with the first three smallest and largest generalized topological indices. MATCH Communications in Mathematical and in Computer Chemistry 2004, 50, 57-62.

38. Ashrafi, A.R.; Ghorbani, M.; Hossein-Zadeh, M.A. The Eccentric Connectivity Polynomial of some Graph Operations. Serdica Journal of Computing 2011, 5, 101-116.

39. Ghorbani, M.; Hosseinzadeh, M.A. A new version of Zagreb indices. Filomat 2012, 26, 93-100, https://doi.org/10.2298/FIL1201093G.

40. Estrada, E.; Torres, L.; Rodriguez, L.; Gutman, I. An atom-bond connectivity index: modelling the enthalpy of formation of alkanes. 1998.

41. Vukičević, D.; Furtula, B. Topological index based on the ratios of geometrical and arithmetical means of end-vertex degrees of edges. J. Math. Chem. 2009, 46, 1369-1376, https://doi.org/10.1007/s10910-0099520-x.

42. Ghorbani, M.; Hosseinzadeh, M.A. Computing ABC4 index of nanostar dendrimers. Optoelectronics and Advanced Materials-Rapid Communications 2010, 4, 1419-1422.

43. Graovac, A.; Ghorbani, M.; Hosseinzadeh, M.A. Computing fifth geometric-arithmetic index for nanostar dendrimers. Journal of Mathematical Nanoscience 2011, 1, 33-42. 